\title{
Lleguar a la Cámara de Diputados de Argentina "desde afuera": recursos, inversiones y marginalidades
}

Artigos Originais

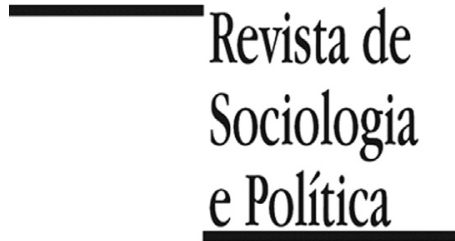

DOI 10.1590/1678-987319277101

\section{Gabriel Levita' \\ Instituto de Problemas Nacionales, Universidad Nacional de Lanús, Consejo Nacional de Investigaciones Científicas y Técnicas (CONICET), UNLa, Lanús, Buenos Aires, Argentina.}

RESUMEN Introducción: El presente artículo indaga desde una sociología política cualitativa las trayectorias sociales y los ingresos a la política partidaria de un conjunto de diputados nacionales argentinos que asumieron sus bancas después de 2007, tras haber acumulado recursos y reconocimiento en otros ámbitos profesionales. Materiales y Métodos: Reconstruimos sus trayectorias laborales, reclutamientos, entradas a la política y llegadas a la Cámara de Diputados a partir de entrevistas en profundidad y de fuentes secundarias. Los casos desarrollados fueron elegidos en función de las posibilidades de cada trayectoria de ofrecer de manera nítida una diversidad de orígenes socioprofesionales y de tipos de capital político. Resultados: El análisis se centra en tres dimensiones: a) la reconversión en capital político de los recursos acumulados en la profesión anterior; b) el ingreso a la competencia partidaria concebido en términos de inversión y riesgo; y c) la posición subalterna de estos actores al llegar al Congreso. Mostramos cómo: a) al momento de la entrada "desde afuera" se aprecian dos tipos de recursos, los individuales y los colectivos; b) los riesgos que asumen al invertir en la política se asocian a la posibilidad de volver al ámbito profesional del que provienen; y c) existe una doble condición de marginalidad, a partir de las posiciones subordinadas que ocupan en la vida parlamentaria y de un extrañamiento respecto al funcionamiento del Congreso. Discusión: El artículo contribuye al conocimiento acerca de las elites políticas argentinas de la actualidad y, específicamente, al de quienes ingresan al Congreso "desde afuera" a partir de la conceptualización de la sociología política francesa en torno a las estructuras de recursos con los cuales estos actores entran a la política. Así, permite conocer las condiciones sociales del reclutamiento político más allá de las variables institucionales.

PALABRAS CLAVE: Elites políticas; Entradas a la política; Trayectorias políticas; Diputados nacionales; Argentina.

Recibido en el 21 de Septiembre de 2018. Aprobado en el 12 de Noviembre de 2018. Acceptado en el 11 de Enero de 2019.

\section{Introducción ${ }^{1}$}

${ }^{1}$ Agradezco las sugerencias y comentarios de Victoria Ortiz de Rozas, Cintia Rodrigo, Luis Donatello, Martín Armelino, Sergio Valenzuela y de los dictaminadores anónimos de la Revista de Sociologia e Política. Una versión preliminar de este trabajo fue presentada en el XIII Congreso de la Sociedad Argentina de Análisis Político, Buenos Aires, 2017.

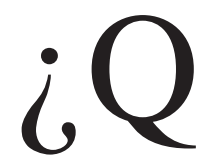

ué significa "ingresar" o "entrar" a la política? Lévêque distingue tres sentidos que se le pueden dar a la expresión. En primer lugar, la socialización política durante la niñez y la infancia implica el acceso a un mundo de significados frente al cual desarrollamos ideas, opiniones y actitudes. Segundo, podemos entrar a la política cuando participamos activamente de una manifestación, cuando votamos o al militar en un partido. Finalmente, entrar a la política puede significar el ejercicio de una función -electiva o no- en alguno de los niveles del Estado o de los partidos (Lévêque 1996). Esta tercera acepción es la que nos interesa aquí para aquellos actores que entran tras haber acumulado recursos y reconocimiento en otros ámbitos profesionales.

En la Argentina, el tema ha sido visto por la opinión pública tanto desde una mirada positiva en términos de "renovación de la política" como, al mismo tiempo, criticado por contribuir a un supuesto deterioro de la calidad de las instituciones. Aunque no se trate de una cuestión novedosa y encontremos este tipo de ingresos ya desde los años 90 , su vigencia parece hacerse cada vez más resonante de la mano de las transformaciones del espacio político argentino de los últimos años. 
2 La Cámara de Diputados tiene 257 miembros elegidos por períodos de cuatro años en representación de cada una de las 23 provincias argentinas y de la Ciudad Autónoma de Buenos Aires. La mitad de las bancas se renuevan cada dos años en los 24 distritos electorales por medio de un sistema de representación proporcional con listas partidarias cerradas regido por el método D'Hondt.

3 El porcentaje de diputados nacionales argentinos que ocuparon sus bancas entre 2003 y 2011 sin contar con militancia partidaria previa y no habiendo ocupado un cargo anterior es de 17,5 , mientras que en los países del Mercosur el promedio es de $18,3 \%$ (Donatello \& Levita 2017).
Estas incluyen una serie de cambios en la matriz bipartidista conformada por el Partido Justicialista (PJ) o peronista y la Unión Cívica Radical (UCR) que había caracterizado a la política nacional desde el retorno al régimen democrático en 1983. A partir de 2001, se produce una fragmentación de los partidos pertenecientes al espacio no peronista y la consiguiente aparición de nuevas fuerzas, más pequeñas, disputándose segmentos del electorado que antes votaba a la UCR (Mustapic 2013; Torre 2003). Una década más tarde, esta fragmentación alcanza también al espacio peronista, cuya fractura en múltiples partidos refleja la heterogeneización de sus bases y arroja a la competencia electoral nuevos contendientes (Torre 2017).

Al mismo tiempo, asistimos a la formación y ascenso del PRO, que reúne a políticos con trayectorias disímiles y que se presenta a sí mismo como un partido outsider, reivindicando el "meterse en la política" por parte de actores que "vienen de afuera" (Vommaro 2017; Vommaro \& Armesto 2015). Aunque no posea mayorías parlamentarias, su crecimiento se manifestó en la última década a través de un arraigo territorial cada vez mayor y de numerosos triunfos electorales que terminaron llevando a la presidencia de la Nación en 2015 a su líder y fundador Mauricio Macri.

También confluyen procesos de más largo aliento como las transformaciones en las lógicas de representación que privilegian la popularidad mediática en el reclutamiento de los candidatos y la personalización de la política (Medina 2004; Manin 1998). Estos cambios promueven un mayor peso de la opinión pública en la selección de candidatos en el marco de instituciones cada vez más débiles (Cheresky 2006; Novaro 2000).

En tal contexto, la pregunta por este fenómeno es también una pregunta acerca de la composición y recomposición de las elites políticas argentinas, su circulación y renovación en el contexto actual. Buscamos, de esta manera, comprender el funcionamiento de la política a partir de estudiar sus fronteras -los ingresos de sus actores- $y$ sus relaciones con otros espacios de poder.

El presente artículo indaga desde una perspectiva cualitativa los rasgos sociológicos de las elites políticas argentinas de la actualidad a partir del análisis de las trayectorias sociales y los ingresos a la política partidaria de un conjunto de diputados nacionales que asumieron sus bancas en los últimos años ${ }^{2} . ¿$ Con que tipo de recursos ingresaron a la política? ¿Cuáles fueron las condiciones de su ingreso a la competencia partidaria? ¿Qué vínculos guardan con sus profesiones anteriores? ¿Cuáles son sus representaciones acerca de la política y de los políticos? Para contestar estos interrogantes nos valemos de fuentes primarias -entrevistas en profundidad- y secundarias, como sus páginas web personales, currículums en línea, bases de datos y artículos de prensa, entre otras.

Analizamos un grupo de actores que llegó a la Cámara de Diputados sin una trayectoria partidaria previa y haciendo valer fundamentalmente los recursos adquiridos en sus recorridos profesionales fuera de los partidos políticos ${ }^{3}$. La selección no fue el azar como en los muestreos estadísticos, sino intencionada en función de las posibilidades de cada trayectoria de ofrecer de manera nítida una diversidad de características sociales y políticas entendidas a partir de las dimensiones analizadas: la reconversión de sus recursos en capital político, su ingreso a la competencia partidaria y sus posiciones dentro de los partidos y en el Congreso. Por ello, el análisis se aboca a descubrir nuevas categorías y sus propiedades sin perseguir una representatividad estadística (Glaser \& Anselm 1967). Nos centramos en las continuidades y rupturas de las trayectorias sociales y las carreras políticas de estos actores, en sus recursos y capitales y en los modos en que estos pueden acumularse, reconvertirse y hacerse valer en situaciones concretas. 
Este artículo se divide en una introducción (I), cinco secciones y unas conclusiones. En la segunda (II) reseñamos las principales categorías formuladas desde las ciencias sociales para el estudio de la problemática en cuestión. En la tercera (III) parte reconstruimos las trayectorias seleccionadas. En el cuarto (IV) apartado analizamos su ingreso a la política partidaria a partir de los recursos y el reconocimiento puestos en valor. La quinta $(\mathrm{V})$ sección estudia esas entradas a la política desde los vínculos entre las profesiones anteriores y la profesión política. La sexta (VI) parte se centra en lo que identificamos como una doble condición de marginalidad relativa de esos actores. Finalmente, las conclusiones (VII) recapitulan los principales puntos del estudio y proponen nuevas hipótesis de trabajo.

\section{Entrar a la política "desde afuera” a partir de las categorías politológicas y sociológicas}

La ciencia política y la sociología han hecho intentos por comprender y explicar el fenómeno, aunque de manera esporádica y poco sistematizada. Desde la primera, se ha privilegiado la categoría de outsiders (Huitt 1961; King 2002) y se ha señalado su creciente número en América Latina (Carreras 2013). Para Linz (1994) se trata de uno de los riesgos del presidencialismo y Barr (2009) señala que todo líder populista es en alguna medida un outsider. Carreras (2012) propone categorías intermedias entre insiders y outsiders tomando en consideración la experiencia política del candidato y el tipo de partido por el que se presenta. Bunker y Navia (2013) reconstruyen una serie de hipótesis vigentes que explicarían el fenómeno de los políticos outsiders en América Latina en los últimos años. Entre ellas destacan un debilitamiento de los clivajes ideológicos que inclinan a los votantes a preferir candidatos no tradicionales; el creciente protagonismo de los perfiles "de gestión" por sobre los políticos habituales y un debilitamiento del sistema de partidos que haría que el electorado se incline por candidatos "antisistema".

Por otro lado, desde la sociología política francesa, el foco ha sido puesto en las entradas al campo político y los capitales con que cada actor ingresa (Bourdieu 1981). Esta mirada ha hecho de la cuestión de la profesionalización uno de sus pilares. De acuerdo al planteo clásico de Weber (2007), el ejercicio de la política como profesión es una actividad de tiempo completo en tanto se vive de ella y para ella. Es decir que garantiza el sustento material al político a condición de que este se aboque a ejercer su profesión política, respectivamente. Ahora bien, Offerlé remarca que entre profesionales y profanos no hay otra cosa que un continuo con diversos tipos de modalidades de dedicación a la política y de financiamiento de las carreras (Offerlé 1996, 2011).

En ese sentido, Gaxie distingue dos tipos de ingresos a la política que se corresponden con dos estructuras del capital político. Primero, se puede ingresar como notable constituyéndose en un empresario político individual, quien es propietario de sus medios de acción y movilización, se relaciona de un modo instrumental con el partido y posee un capital muy ligado a su persona, que no tiene origen en el campo político. Por otra parte, el que ingresa como militante es representante de una empresa política colectiva, es decir que se apoya en los recursos organizacionales colectivos del partido. El autor distingue una tercera forma de ingresar a la política, como protegido o colaborador de un líder, combinando sus propios recursos con las posibilidades que les brindan los dirigentes de un partido (Gaxie 2004; Offerlé 2004).

Las entradas al campo político pueden realizarse a partir de estrategias de reconversión en las que distintos tipos de recursos se transforman en capital político (Joignant 2012; Tissot 2004). Asimismo, Boltanski sostiene a partir de la idea de multiposicionalidad que los actores dominantes en un campo también tenderán a ocupar las posiciones más privilegiadas en los otros en que participe 
(Boltanski 1973). Esta interdependencia entre la política y otros espacios obliga a reparar en que, en todos los casos, los factores extra-políticos son centrales en la explicación de las carreras políticas (Gaxie 2002).

En la Argentina, los ingresos a la competencia partidaria y el reclutamiento político durante los últimos años han sido estudiados desde la sociología tanto para el nivel legislativo (Canelo 2011; Landau 2015; Levita 2015; Mellado 2016; Rodrigo 2014; Rozas 2011) como para los gabinetes y los poderes ejecutivos en general (Heredia, Gené \& Perelmiter 2012; Perelmiter 2012; Giorgi 2014; Canelo 2019; Gené 2019; Sosa 2019). Estos trabajos destacan una variedad de recursos movilizados para ingresar a la actividad partidaria, tales como los educativos, militantes, sindicales, de experticia y mediáticos, entre otros. Asimismo, subrayan el peso de las sociabilidades familiares, religiosas, escolares y militantes como vías de acceso a cargos electivos o a la función pública.

El análisis de las trayectorias que entran "desde afuera" ha quedado más relegado y ha sido trabajado principalmente en relación a figuras protagónicas de la escena nacional (Lascurain 2018) y a propósito de la división del trabajo político entre "nuevos" y "viejos" cuadros del PRO (Vommaro 2017; Vommaro \& Armesto 2015). En la región, otros trabajos se han formulado la pregunta centrándose por caso tanto en las entradas al parlamento brasilero (Bolognesi, Perissinotto \& Codato, 2019; Junior \& Filho 2012; Santos 1997).

Desde el punto de vista metodológico, el estudio del reclutamiento político demanda la integración de una pluralidad de variables tales como el diseño institucional, las culturas organizativas y las oportunidades coyunturales junto con otras como las trayectorias, redes y sociabilidades (Sawicki 1999). A su vez, los trabajos sobre reconversiones políticas se encuentran muy ligados a los estudios de trayectorias y redes sociales (Fillieule 2001). Éstos han sido utilizados para describir y explicar fenómenos y procesos cuyos rasgos sólo pueden ser entendidos en relación a las experiencias y recorridos de los actores que participan en ellos (Cucchetti 2010; Vázquez 2014).

\section{Trayectorias profesionales y reclutamiento político}

El periodismo ya ha sido señalado como potencial ámbito de reclutamiento para las candidaturas a puestos legislativos (Dogan 1998). En el congreso nacional argentino, algunos de los casos más significativos han sido los de María América González - diputada por la Ciudad de Buenos Aires primero por el FrePaSo y luego por el ARI durante tres períodos consecutivos a partir de 1997-, Norma Morandini-diputada por Córdoba por el Frente Cívico en 2005 y luego senadora-, María Eugenia Estenssoro -senadora por la Ciudad de Buenos Aires por la Coalición Cívica-ARI en 2007 y antes legisladora- o Alejandro Grandinetti -electo diputado por Santa Fe por UNA en 2015-, entre otros.

${ }^{4}$ Fuentes para el desarrollo del caso: entrevista realizada en el despacho de la diputada en octubre de 2016, ficha de Directorio Legislativo, página web personal.
Cuando tenía 23 años Mirta Tundis 4 (La Matanza, Buenos Aires 1956) comenzó a trabajar en las cajas jubilatorias, atendiendo al público y encargándose del seguimiento de los trámites jubilatorios de los aportantes. Luego fue pasando por varias posiciones hasta llegar a ser secretaria del director nacional de Cajas de Industria y Comercio. Hacia finales de los años ochenta y comienzos de los noventa pasó por distintos cargos entre la obra social de los jubilados (PAMI), la Secretaría de Seguridad Social y la Administración Nacional de Seguridad Social (ANSES) de la mano del entonces secretario del área, Santiago de Estrada, a quien había conocido cuando éste ocupaba el mismo cargo el año de su ingreso.

De Estrada escribía además una columna en el matutino Clarín y tenía relación con varios periodistas de ese medio, quienes también trabajaban en Ca- 
${ }^{5}$ Durante los gobiernos de Néstor y Cristina Kirchner, Sergio Massa fue director ejecutivo de la ANSES y jefe de gabinete. Tras las elecciones de 2009 comienza a separarse de ese espacio político y para los comicios legislativos de 2013 conforma el Frente Renovador, de orientación peronista y constituido a partir de su liderazgo sobre un conjunto de intendentes de la provincia de Buenos Aires.

\footnotetext{
${ }^{6}$ Fuentes para el desarrollo del caso: entrevista realizada en el despacho del diputado en septiembre de 2016, ficha de Directorio Legislativo, entrevista a la prensa (El Gráfico 2012), autobiografía (Baldassi 2016).
}

nal 13, propiedad del mismo grupo empresario. El entonces director de noticias del canal, convocó a través suyo a Mirta para realizar una participación sobre temas previsionales en el noticiero del mediodía. Al tiempo, esta se transformaría en una columna periódica y en un espacio fuera del estudio, el Mirta móvil. Con la creación del canal de cable Todo Noticias (TN), comenzó a conducir el programa Jóvenes de la tercera edad y, años más tarde, Mirta te acompaña en el canal Metro. A lo largo de la década del 2000 continuó en los noticieros de Canal 13 y TN, además de participar en diversos programas de Radio Mitre.

Para las elecciones 2003, a Tundis ya le habían ofrecido ingresar en las listas de candidatos para legisladores de la Ciudad Autónoma de Buenos Aires por los partidos de Patricia Bullrich y de Santiago de Estrada, quien apoyaba la candidatura de Mauricio Macri a Jefe de Gobierno con una lista de legisladores encabezada por él mismo. Pero no sería hasta 2013 cuando acepta la oferta de Sergio Massa, a quien había conocido cuando este era director ejecutivo de la ANSES entre 2002 y 2007. Massa le ofrecía integrar la lista de candidatos a diputados nacionales por la provincia de Buenos Aires del Frente Renovador, que encabezaba él mismo tras su ruptura con el gobierno de Cristina Kirchner. Estas fueron las primeras elecciones en que compitió con su nueva fuerza ${ }^{5}$.

Tundis quedo en el tercer puesto de la lista de candidatos y, tras el resultado favorable en las elecciones legislativas de 2013, asumió como diputada nacional en diciembre de ese año. Dos años después, fue candidata a vice jefa de gobierno de la Ciudad de Buenos Aires acompañando a Guillermo Nielsen en la lista del Frente Renovador. La fórmula no obtuvo los votos mínimos necesarios en las primarias y no pudo competir en la elección general. En 2017 resulta reelecta en el segundo puesto de la lista de diputados del massismo bonaerense.

La entrada a la política partidaria desde el mundo del deporte puede apreciarse tanto en cargos electivos como no electivos (Bart 2011). En la Argentina resulta ineludible la figura del ex automovilista Carlos Reutemann, quien comenzó su carrera política en 1995 y fue electo gobernador por el justicialismo de Santa Fe en dos oportunidades y senador nacional en cuatro (Lascurain 2018). El congreso argentino también registra en años recientes al ex futbolista Carlos Mac Allister -diputado por La Pampa por el PRO en 2013 y luego Secretario de Deportes de la Nación-, al ex tenista Agustín Calleri -diputado por Córdoba por el massismo en 2013- y, más atrás, al ex futbolista Antonio Rattín - diputado por la provincia de Buenos Aires en 2001 y luego concejal de Vicente López-, entre otros. Desde el arbitraje, el caso más conocido es el de Javier Castrilli, funcionario del área de deportes de la provincia de Buenos Aires en 2000 y más tarde candidato fallido a Jefe de Gobierno de Buenos Aires y a concejal de La Matanza.

Héctor Baldassi ${ }^{6}$ (Río Ceballos, Córdoba 1966) cursó dos años de la carrera de medicina en la Universidad Nacional de Córdoba hasta que se fue a vivir a Buenos Aires, en donde entró a trabajar en una empresa metalúrgica. En el año 1989 comenzó el curso de arbitraje de la Asociación del Fútbol Argentino (AFA), dando inicio a su carrera como réferi, que lo llevaría a lo largo de la década del noventa por las distintas divisiones del fútbol argentino hasta debutar en primera en 1999. Simultáneamente, fue repartidor de herramientas para talleres mecánicos y luego se desempeñó como escritor de espacios publicitarios en distintos medios gráficos. Trabajó en un diario sindical y realizó asesorías en organización de eventos.

Con el paso al arbitraje internacional en la década del 2000, se profesionalizó pudiendo vivir únicamente de ese trabajo. Dirigió partidos de primera división, copas internacionales, eliminatorias, los juegos olímpicos en 2008 y el mundial en 2010. Arbitró su último partido a fines de 2011 tras lo cual comenzó 
${ }^{7}$ Cambiemos es la alianza política conformada en 2015 por el PRO, la Coalición Cívica y el sector mayoritario de la Unión Cívica Radical. Llevó como candidato presidencial al líder y fundador del PRO, Mauricio Macri, quien derrotó al postulante kirchnerista en las elecciones de ese año. ${ }^{8}$ Fuentes para el desarrollo del caso: entrevista realizada en el despacho del diputado en octubre de 2016, ficha de Directorio Legislativo, página web personal.

\footnotetext{
${ }^{9}$ Desde 2004, el fiscal federal Alberto Nisman estaba a cargo de la investigación del atentado terrorista contra la sede de la AMIA, que tuvo lugar en la Ciudad de Buenos Aires en 1994. En enero de 2015 denunció a la entonces presidenta Kirchner y a funcionarios de su gobierno por supuesto encubrimiento a los principales sospechosos en complicidad con el gobierno iraní.

${ }^{10}$ Un día antes de exponer su denuncia en el Congreso Nacional, Nisman fue hallado muerto. El gobierno sostuvo la tesis del suicidio, mientras que los principales dirigentes opositores avalaron la hipótesis del asesinato.
}

a trabajar haciendo las relaciones públicas de la AFA en los partidos de las eliminatorias, por un ofrecimiento del entonces presidente de la institución Julio Grondona.

A fines de 2012 el periodista Fernando Niembro -quien fuera candidato a diputado nacional del PRO por la provincia de Buenos Aires hasta que renunció por denuncias de corrupción en su contra- le propuso integrar la lista de candidatos a diputados nacionales por Córdoba para las elecciones legislativas del año siguiente. Tras hablarlo con otros dirigentes partidarios como Emilio Monzó y con el propio Mauricio Macri, Baldassi aceptó ser el primero en la lista.

Acepta la candidatura y, tras quedar en cuarto puesto en las elecciones, asume como diputado en diciembre de 2013 con mandato hasta 2017. En 2015 fue candidato a vicegobernador de Córdoba junto a Oscar Aguad por la alianza Cambiemos $^{7}$, quedando en segundo lugar. En 2017 es reelegido como primer diputado de Cambiemos por Córdoba.

Con el antecedente del rabino Sergio Bergman y su ingreso a la política desde la dirigencia comunitaria judía -fue legislador de la Ciudad de Buenos Aires por el PRO en 2011, diputado nacional en 2013 y ministro de Ambiente y Desarrollo Sustentable a partir de 2015-, podemos analizar el caso de Waldo Wolff $^{8}$ (Buenos Aires 1968). Comenzó de adolescente siendo arquero de fútbol en el Club Náutico Hacoaj. Durante sus estudios universitarios en administración de empresas en la Universidad de Belgrano jugó en diversos clubes argentinos y, más tarde, un año en Israel. Al volver al país a fines de la década del noventa se incorporó a la empresa familiar dedicada a negocios inmobiliarios y a desarrollos en la construcción de edificios en la zona norte de la Capital y el Gran Buenos Aires. Al mismo tiempo, cursó una maestría en negocios internacionales y se recibió de martillero público.

Paralelamente, fue dirigente comunitario en numerosas instituciones de la comunidad judía local y vicepresidente de la DAIA (institución a cargo de la representación política de las organizaciones judías argentinas). Desde este último cargo trabó una relación de cercanía con el fiscal Nisman en el marco de la investigación que este último conducía sobre el atentado a la sede de la AMIA $\mathrm{y}$, posteriormente, de su denuncia contra funcionarios del gobierno por el supuesto encubrimiento de los responsables ${ }^{9}$. Con la muerte del fiscal en 2015, Wolff adquirió cierto protagonismo mediático oponiéndose al entonces gobierno de Cristina Kirchner y al modo en que había tratado política y judicialmente la cuestión ${ }^{10}$. En ese contexto, participó en programas periodísticos, hizo público el hecho de recibir una serie de amenazas y fue denunciado por traición a la patria.

En 2015 Jorge Macri - primo de Mauricio y entonces intendente de Vicente López y jefe de campaña del PRO en la provincia de Buenos Aires- le propone formar parte de la lista de diputados nacionales del PRO en esa provincia. Se conocían a partir de las distintas actividades que la DAIA organizaba en el municipio, como presentaciones de libros o informes.

Acordó formar parte de la lista en el décimo lugar, con pocas expectativas de ingresar a la Cámara. Sin embargo, gracias al triunfo del PRO en la provincia obtuvieron una cantidad inesperada de bancas y Wolff asumió como diputado en diciembre de 2015 con mandato hasta 2019. Cuatro años después resultó reelegido hasta 2023.

Otro ámbito de ingreso es de los movimientos de trabajadores desocupados y de piqueteros, que tuvieron su auge a fines de la década del noventa y comienzos de los dos mil con la profundización del desempleo y la pobreza. No obstante, se dio un desfasaje entre la capacidad de movilización de esas 
${ }^{11}$ Fuentes para el desarrollo del caso: entrevista realizada en la cooperativa La Juanita en septiembre de 2016, ficha de Directorio Legislativo, artículos de prensa (La Nación 2015; El Cronista 2015). organizaciones y los espacios de poder institucionalizados a los que llegaron sus dirigentes. Estos fueron pocos, mayormente en el nivel ejecutivo y de segunda o tercera línea (Massetti 2011). Uno de los dirigentes más relevantes, Luis D’Elía de la FTV llegó a concejal por La Matanza, diputado provincial y luego Subsecretario de Tierras en la gestión kirchnerista. En el congreso nacional encontramos el caso de Néstor Pitrola del Polo Obrero, elegido diputado en 2013 y en 2015.

Por su parte, Héctor "Toty" Flores" (San José de Feliciano, Entre Ríos 1953) llegó desde su pueblo natal al Gran Buenos Aires a los 18 años y se fue a vivir a una villa de emergencia en el barrio de Loma Hermosa. Comenzó a trabajar como obrero metalúrgico en hasta que fue despedido en 1993 e instaló por su cuenta un pequeño taller de costura.

Durante sus años en las fábricas se acercó compañeros sindicalistas, aunque sin participar formalmente en ningún partido u organización. Con la vuelta a la democracia en 1983 militó un tiempo en el Movimiento al Socialismo (MAS). Durante su emprendimiento como textil continuó vinculado con ex metalúrgicos con quienes comenzó a involucrarse en los primeros piquetes en protesta por el desempleo. De esa experiencia surgió a mediados de los noventa el Movimiento de Trabajadores Desocupados de La Matanza, del que Toty fue un activo participante. Hacia finales de la década, junto a un grupo de compañeros del MTD fundaron en su barrio en Laferrere la cooperativa La Juanita, que con el tiempo abriría un jardín de infantes, un taller de costura y una panadería.

Al comenzar la cooperativa, el grupo decidió no recibir aportes estatales a través de subsidios o planes sociales, sino generar sus propios recursos a partir de microemprendimientos como la panadería y el taller textil. Esta particularidad los distinguió de otras organizaciones de desocupados y fue la causa de que el entonces director de la ONG Poder Ciudadano se acercara para proponerles proyectos conjuntos. Organizaron cursos sobre política y formación ciudadana.

En este marco, comenzaron a relacionarse con dirigentes políticos opositores al gobierno de entonces, que estaban interesados en vincularse con una agrupación que rechazaba los planes sociales del Estado. También entraron en relación con ONG y empresarios privados que le fueron facilitando maquinaria, materias primas y know how para la producción de diversos artículos que daban trabajo a los socios y recursos para las otras actividades. A su vez, esta particular relación con el Estado y con el sector privado le dio cierta notoriedad mediática e hizo que el funcionamiento de La Juanita fuese puesto como modelo por los actores que la patrocinaban, contraponiéndola a otros grupos que se habían acercado al kirchnerismo y recibían recursos estatales.

En 2006 trabó conocimiento con la política opositora Elisa Carrió, quien había ido a La Juanita a dar una charla sobre la problemática de la drogadicción y el narcotráfico. Al año siguiente, esta le ofrece formar parte de la lista de diputados nacionales por la provincia de Buenos Aires de su flamante partido Coalición Cívica (CC).

El primer ofrecimiento fue de encabezar la lista, pero conforme avanzaron las negociaciones entre los distintos sectores de la coalición, terminó en el puesto sexto. Si bien el interlocutor de Carrió era Toty, con quien además fue trabando una relación personal, las decisiones que éste tomaba eran consensuadas con los miembros de la cooperativa. Finalmente, la CC tuvo un muy buen desempeño en las elecciones e ingresaron nueve diputados. Toty asumió su banca en diciembre de 2007 con mandato hasta 2011. Ese año fue candidato a senador nacional por la provincia de Buenos Aires, quedando en séptimo lugar. Al año siguiente fundó junto a Carrió el Movimiento Humanista de Resistencia 
y Construcción 2012 y en 2013 el Movimiento Social por la República. Fue precandidato a vicepresidente acompañando a Carrió en las primarias de Cambiemos en 2015, de las que resultaría la fórmula presidencial Macri-Michetti. A comienzos de 2016 fue funcionario municipal durante tres meses de la flamante gestión de Cambiemos en Mar del Plata. En 2017 es electo como diputado nacional en segundo lugar por Cambiemos en la provincia de Buenos Aires (Cuadro 1).

Cuadro 1 - Síntesis de los cuatro casos

\begin{tabular}{|c|c|c|c|c|}
\hline Diputados & Tundis & Baldassi & Wolff & Flores \\
\hline $\begin{array}{l}\text { Principales profesiones } \\
\text { ejercidas con } \\
\text { anterioridad }\end{array}$ & $\begin{array}{l}\text { Empleada en el } \\
\text { PAMI, periodista }\end{array}$ & Árbitro de fútbol & $\begin{array}{l}\text { Empresario, dirigente } \\
\text { de la comunidad judía }\end{array}$ & $\begin{array}{l}\text { Dirigente de } \\
\text { MTD, referente de } \\
\text { cooperativa de trabajo }\end{array}$ \\
\hline $\begin{array}{l}\text { Situación al momento } \\
\text { del reclutamiento }\end{array}$ & $\begin{array}{l}\text { Periodista en Canal } \\
\text { 13, TN, Metro y Radio } \\
\text { Mitre }\end{array}$ & RRPP de la AFA & $\begin{array}{l}\text { Empresario } \\
\text { inmobiliario y de la } \\
\text { construcción, vicepresi } \\
\text { dente de la DAIA }\end{array}$ & $\begin{array}{l}\text { Referente de la } \\
\text { cooperativa La Juanita }\end{array}$ \\
\hline $\begin{array}{l}\text { Conocimiento } \\
\text { mediático }\end{array}$ & $\begin{array}{l}\text { Amplio desde hace más } \\
\text { de } 20 \text { años }\end{array}$ & $\begin{array}{l}\text { Amplio desde hace más } \\
\text { de } 20 \text { años }\end{array}$ & $\begin{array}{l}\text { Restringido y muy } \\
\text { reciente, focalizado en } \\
\text { el caso Nisman }\end{array}$ & $\begin{array}{l}\text { Restringido y secto a } \\
\text { partir de La Juanita y } \\
\text { de su entrada en } \\
\text { política }\end{array}$ \\
\hline Reclutador & Sergio Massa & Fernando Niembro & Jorge Macri & Elisa Carrió \\
\hline Partido & Frente Renovador & PRO - Cambiemos & PRO - Cambiemos & $\begin{array}{l}\text { Coalición Cívica / CC } \\
\text { - Cambiemos }\end{array}$ \\
\hline Elecciones anteriores & $\begin{array}{l}\text { DN } 2013 \text { (ganadas) } \\
\text { VJG } 2015 \text { (perdidas en } \\
\text { PASO) DN } 2017 \\
\text { (ganadas) }\end{array}$ & $\begin{array}{l}\text { DN } 2013 \text { (ganadas) VG } \\
2015 \text { (perdidas) DN } \\
2017 \text { (ganadas) }\end{array}$ & DN 2015 (ganadas) & $\begin{array}{l}\text { DN } 2007 \text { (ganadas) SN } \\
2011 \text { (perdidas) VP } \\
2015 \text { (perdidas en } \\
\text { PASO) DN } 2017 \\
\text { (ganadas) }\end{array}$ \\
\hline Mandato $N^{o} 1$ & DN 2013-2017 & DN 2013-2017 & DN 2015-2019 & DN 2007-2011 \\
\hline Lugar en la lista & $3^{\circ}$ & $1^{\circ}$ & $11^{\circ}$ & $6^{\circ}$ \\
\hline $\begin{array}{l}\text { Cargos de autoridad en } \\
\text { comisiones al inicio del } \\
\text { mandato } N^{o} 1\end{array}$ & $\begin{array}{l}\text { Personas Mayores } \\
\text { (Presidenta) }\end{array}$ & $\begin{array}{l}\text { Deportes } \\
\left(\text { Vicepresidente } 1^{\circ} \text { ) }\right.\end{array}$ & - & $\begin{array}{l}\text { Prevención de } \\
\text { Adicciones y Control } \\
\text { del Narcotráfico } \\
\text { (Secretario) }\end{array}$ \\
\hline Mandato $N^{o} 2$ & DN 2017-2021 & DN 2017-2021 & DN 2019-2023 & DN 2017-2021 \\
\hline Lugar en la lista & $2^{\circ}$ & $1^{\mathrm{o}}$ & - & $2^{\circ}$ \\
\hline $\begin{array}{l}\text { Cargos de autoridad en } \\
\text { comisiones al inicio del } \\
\text { mandato } N^{o} 2\end{array}$ & $\begin{array}{l}\text { Personas Mayores } \\
\text { (Presidenta) }\end{array}$ & $\begin{array}{l}\text { Deportes } \\
\left(\text { Vicepresidente } 1^{\circ} \text { ) }\right.\end{array}$ & $\begin{array}{l}\text { Bicameral de } \\
\text { fiscalización de los } \\
\text { órganos y actividades } \\
\text { de seguridad interior. } \\
\text { (Presidente) }\end{array}$ & $\begin{array}{l}\text { Derechos Humanos } \\
\text { (Presidente) }\end{array}$ \\
\hline $\begin{array}{l}\text { Experiencia previa en } \\
\text { actividades de } \\
\text { representación }\end{array}$ & No & No & $\begin{array}{l}\text { Sí, como dirigente } \\
\text { comunitario }\end{array}$ & $\begin{array}{l}\text { Sí, como dirigente de } \\
\text { movimientos sociales }\end{array}$ \\
\hline $\begin{array}{l}\text { Posibilidad de } \\
\text { continuar o volver a su } \\
\text { actividad precedente }\end{array}$ & $\begin{array}{l}\text { Continúa con un } \\
\text { programa de cable y } \\
\text { tiene ofertas para } \\
\text { volver plenamente a los } \\
\text { medios }\end{array}$ & No como árbitro & $\begin{array}{l}\text { Continúa como } \\
\text { empresario. Ciclo en la } \\
\text { dirigencia comunitaria } \\
\text { "agotado" }\end{array}$ & $\begin{array}{l}\text { Continúa como } \\
\text { referente de la } \\
\text { cooperativa }\end{array}$ \\
\hline
\end{tabular}

Fuente: el autor.

Obs.: Abreviaturas de otros cargos: VP: Vicepresidente; VG: Vicegobernador; SN: Senador nacional; DN: Diputado nacional; VJG: Vicejefe de gobierno. 


\section{La reconversión de recursos en capital político}

¿Con qué tipo de recursos se ingresa a la competencia política cuando no existe una trayectoria previa de militancia en la fuerza por la cual el actor se presenta ni en ningún otro partido político? En un nivel más amplio, a la pregunta por las estructuras de los capitales políticos de los actores, la sociología política responde con la distinción típica ideal ya clásica entre los recursos propios y los recursos obtenidos en base al partido o a otra organización colectiva (Gaxie 2004). Es decir, entre la posesión de un capital ligado a la persona y otro derivado de la pertenencia a un colectivo. Así, podremos encontrar políticos con estructuras de capitales más individuales o más colectivas en función de si deben su ascenso a sus propios medios o a los del partido, respectivamente. Empíricamente, estos dos tipos pueden darse de manera combinada.

Ahora bien, quienes ingresan en la competencia política partidaria sin militancia partidaria y habiendo desarrollado carreras profesionales por fuera de la política institucionalizada, lo hacen con determinados recursos materiales y simbólicos, que, recuperando el concepto de capital, bien podemos llamar capitales extrapolíticos. Éstos se movilizan en la arena política reconvirtiéndose, eventualmente, en capitales políticos.

También aquí es posible distinguir estructuras de capitales extrapolíticos más individuales o más colectivas. El primer tipo corresponde a aquellos que han construido su reconocimiento en base a su figura personal y a su trayectoria individual, mientras que el segundo es el de quienes deben su capital al hecho de ser o haber sido representantes de una organización o un grupo más o menos constituido y deben su valoración al colectivo al que pertenecen, más allá de que actúen o no en nombre de este.

Un primer tipo de capital extrapolítico proviene entonces del reconocimiento público de un cierto prestigio personal originado en la actividad profesional de la que participan. Entendido por los actores en términos de credibilidad, honestidad, experiencia, habilidad, virtud, etc., pueden originarse en distintos ámbitos como el periodismo, el deporte, el arte o el espectáculo y tienen una de sus condiciones de posibilidad en tratarse de personajes con presencia en los medios de comunicación.

Mi temor era o sea, yo construí durante muchísimos años el hecho de que la gente
crea en mí. Ser genuina. Si me equivocaba, si daba una información que era
incorrecta al día siguiente aceptar que me había equivocado, pero siempre decir
la verdad, porque era mi único capital. Imaginate perder ese capital que vos du-
rante tantos años formaste. Que la gente crea en vos, que te crea genuina,
honesta. Era lo que pensé que iba a perder [...]. Así que me costaba mucho pensar
qué iban a decir de diputada, corrupta, este la verdad que en el momento que
tome la decisión sufrí muchísimo (Tundis, entrevista).

El ser percibido por el público como alguien que dice la verdad y reconoce sus errores aparece como un elemento central en la valoración pública de la periodista. Esta percepción se encuentra de algún modo objetivada en su trayectoria profesional y en su permanencia en los medios de comunicación en los que trabaja. También el referato deportivo requiere un grado de reconocimiento que puede verse asimismo en el recorrido de crecimiento profesional de Baldassi y su llegada a las instancias superiores del arbitraje internacional.

La gente te conoce y sabe lo que vos fuiste, y la gente te respeta por lo que fuiste. Yo siempre sostuve esto: yo me gané un pequeño prestigio, ese prestigio no lo voy a perder. ¿Cómo no se pierde? Trabajando, luchando, seguir caminando, seguir siendo lo que vos fuiste a través de ese prestigio que te permitió estar en otro lugar y el reconocimiento por parte de la gente (Baldassi entrevista). 
Un segundo tipo de capital extrapolítico podemos definirlo como colectivo, en tanto el reconocimiento del que es portador el actor se origina en los recursos de una organización a la que pertenecen o pertenecieron y que, en algunos casos, es la que se pone en valor a través del actor en cuestión. Su posición se la deben al grupo o institución de la que provienen, como puede ser el caso de los sindicalistas, los miembros de organizaciones patronales, los integrantes de ONG y los representantes de movimientos sociales, entre otros, más allá de que al momento de ser reclutados se encuentren o no vinculados a dicha organización.

Nosotros éramos un símbolo en el sentido que habíamos rechazado los planes sociales y eso era lo que más impacto tenia. [...] Éramos conocidos por eso, eso era nuestra cédula de identidad. [...] Yo creo que aparecía como algo raro esto de haber rechazado los planes sociales. Nosotros cuando lo hicimos no nos dimos cuenta que apuntamos al corazón del clientelismo político. Los que estaban afuera que tenían una visión más clara veían que era muy simbólico esto que nosotros sobreviviéramos, éramos sobrevivientes de ese proceso y creo que generaba de alguna manera una empatía con estos sectores que quisieran ver un país así (Flores entrevista).

En el caso de Flores, este peso preponderante de lo colectivo se ve, en primer lugar, en el hecho de que las decisiones de participar o no, como vimos en el apartado anterior, son sometidas a algún grado de discusión con los dirigentes de su movimiento. Pero lo central es que aquello que se le reconoce como valor es producto de la organización en la que participa y no de cualidades estrictamente personales. En este mismo sentido, el caso de Wolff también es el de alguien que debe su posición frente a la política a su rol de dirigente comunitario. Difiere de Flores en que no se desempeña como dirigente de ninguna institución en particular al momento de ser diputado y no se candidateó a la banca en tanto tal. Sin embargo, su reconocimiento público, su papel en la cuestión del fiscal Nisman son producto de una acumulación como dirigente comunitario, especialmente como vicepresidente de la DAIA.

Otra característica de quienes han acumulado en organizaciones y colectivos es que poseen experiencia en actividades de representación política. Es decir, poseen una serie de saberes y dominan un conjunto de prácticas sobre la relación entre los representantes y los representados, la formación de alianzas, la competencia en elecciones internas, la negociación, la construcción de consensos, etc. Este saber hacer representativo es propio de quienes han construido su reconocimiento de manera colectiva y supone una condición diferente en el ingreso a la política partidaria.

A mí me dio mucha ventaja haber manejado seis años una federación que tiene
42.000 afiliados, 80.000 afiliados indirectos. Porque lo primero que tenés que
entender cuando vos tomas decisiones políticas como representante de espacios
tan heterogéneos es que muchas veces vos estas convencido de que estás
haciendo lo que corresponde o lo mejor para el promedio y todos te putean. [...].
Entonces muchas veces a mí me pasaba en DAIA. Lógico, cada vez que tomas
una decisión la mitad te putea y la otra mitad no. O te putean todos. [...] Vos
pensá que muchos llegan a diputados sin haber tenido o habiendo tenido mucha
menos gestión que yo a nivel de representatividad y análisis de situaciones de
conflicto y análisis de tomas de decisiones. Yo tengo una probeta de ensayo
espectacular. Además, en un universo complejísimo (Wolff entrevista).

Estos dos tipos de capital extrapolítico, el individual y el colectivo, no son en modo alguno excluyentes. De hecho, todo político tiene algún grado de ambos, aunque, generalmente, en distintas proporciones. Los cuatro actores que analizamos en este trabajo permiten ver con claridad las características de cada tipo, apreciando también las diferencias y complejidades de cada caso. La reconversión de estos recursos, a su vez, no va de suyo, sino que requiere de la 
existencia de redes de contactos y relaciones que posibilitan una candidatura en situaciones concretas (Mathieu 2007).

A los recursos construidos fuera de la política partidaria podemos sumarle en la valoración de estos actores a la hora de ser reclutados lo que Bruère y Gaxie (2014) denominan "representación simbólica". Cada uno de ellos puede ser visto a partir de su consideración pública como representante de un sector en las listas de candidatos. Por ejemplo, una candidata "que viene del" periodismo o que conoce de temas de jubilados, un candidato "que viene del" deporte, otro de la comunidad judía o cercano al fiscal Nisman, otro de los movimientos sociales y las cooperativas.

\section{Diversificando riesgos: No toda inversión es absoluta}

Aceptar una candidatura para diputado puede ser visto como una apuesta en la que se consiente en invertir una determinada cantidad de recursos, tiempo, esfuerzo, trabajo, emociones y expectativas. Si se gana el cargo para el cual se postuló, las actividades profesionales que se ejercían antes de asumir se verán en algún modo postergadas. Ya sea porque no se podrán realizar más por falta de tiempo y capacidad o porque se les destinará mucha menos atención y espacio en la agenda. De allí que toda inversión en la competencia partidaria pueda ser vista al mismo tiempo como una desinversión en el espacio del cual proviene el actor.

Esta operación comporta sus riesgos y suscita, más o menos conscientemente, evaluaciones por parte del actor: ¿Puedo seguir creciendo o, al menos, conservar mi posición en la actividad profesional a la que me dedico? ¿Cuánto estoy dispuesto a postergarla en pos de la aventura política? ¿Puedo seguir vinculado a esta al tiempo que ocupo un cargo político? Si fracaso en mi candidatura o no resulto reelecto ¿hasta qué punto podré volver a mi posición anterior?

Weber ya apuntaba que existen diversas maneras de vincularse con la política como profesión, teniéndola a esta como actividad principal o secundaria (Weber 2007). Aquí las particularidades de cada trayectoria se combinan con las características de los distintos ámbitos profesionales en que cada uno se desempeñaba con anterioridad. Así, el mundo del periodismo en los medios masivos de comunicación permite no sólo continuar teniendo un programa de televisión mientras se es diputado -aunque signifique menos horas frente a las cámaras y micrófonos que antes de asumir el cargo-, sino que también supone la posibilidad siempre latente de volver a la posición anterior sin grandes costos.

Voy a seguir con mi programa de cable, que lo tengo y eso me da mucha satisfacción. Y por ahí continuaré Muchos en los medos me dicen "volvé con nosotros". Tengo el programa, pero también podría hacer radio (Tundis entrevista).

Esta vinculación con la profesión anterior y la posibilidad de volver a ella en caso de no poder renovar su banca, perder una elección o quedarse sin ningún cargo no se relaciona directamente con el peso de lo individual o lo colectivo en el capital de cada político. Se vincula antes bien con el tipo de actividad realizada y con el recorrido particular de cada uno. En ese sentido, este rasgo parece compartido por actores con recorridos tan disímiles como Tundis y Flores.

Yo sabía que después iba a volver acá [a La Juanita]. Esto yo creo también que es tranquilizador, sabes que tenés un lugar donde volver. No te obsesionás por estar ahí, no, no. No pasa nada, viste. Yo creo que eso fue el gran desafío, que yo ya lo probé, que es bueno tener un lugar donde volver. Por supuesto que a mí me gustaría hoy participar en la política, por supuesto que es parte de mi vida, quiero 
hacerlo, quiero estar en un lugar donde pueda construir, tener posibilidades, donde pueda trabajar con la gente y toda la historia. Pero no la obsesión, no pasa nada si no estoy. Tengo libertad de elegir (Flores entrevista).

Flores continuó como dirigente de la cooperativa durante su mandato de diputado y siguió siéndolo al terminar el mismo. Presumiblemente, durante los cuatro años que duró su banca tuvo que relegar en alguna medida parte de su papel como máxima autoridad, pero sin consecuencias mayores a la hora de retomar nuevamente de manera plena su rol en La Juanita. Esa posibilidad de volver es esgrimida como un valor positivo, ya que no dependería exclusivamente de la política para subsistir y puede escoger si presentarse o no a las elecciones.

El caso de Wolff hace necesario reparar en dos espacios distintos. En tanto empresario inmobiliario y de la construcción, conserva su participación en la firma familiar y continúa desempeñando esa actividad mientras es diputado. En este sentido, no hay una renuncia a dicho rol, aun cuando el mismo pueda verse perjudicado por el tiempo que insume su trabajo como diputado.

No hago esto [la política] por dinero. Al revés, pierdo plata por estar acá. Tengo una actividad privada [la empresa constructora] que, de hecho, la descuido bastante (Wolff entrevista).

Ese relegar dinero por hacer política es resignificado positivamente como un sacrificio. Ahora bien, en tanto dirigente comunitario, en tanto especialista de la representación, percibía sus posibilidades de continuar como limitadas. La reconversión de ese capital en capital político y la apuesta que eso significa adquiere un sentido ante el "agotamiento" de su rol en la dirigencia comunitaria.

Fue una apuesta muy grosa porque yo terminé ocupando el puesto número doce en la lista de diputados provinciales. Y para que yo entrase Cambiemos tenía que hacer la mejor elección, que terminó siendo en la historia para que entren doce diputados. Y muchos me decían "vos estás loco porque sos candidato a presidente de la DAIA natural y te jugas a ser diputado nacional cuando es casi imposible que lo seas". Y yo tomé la decisión basada en principios, porque entendí que mi paso por la dirigencia comunitaria estaba absolutamente agotado (Wolff entrevista).

Diferente es la situación en el mundo del deporte, tanto para los jugadores como para los árbitros. La carrera del deportista termina relativamente rápido en la duración de la vida de una persona, si bien la edad a la que se deja de jugar varía en los distintos deportes. En cuanto a los réferis de fútbol, a diferencia de los jueces de otras disciplinas, requieren un entrenamiento físico importante para correr por toda la cancha siguiendo las jugadas de cerca durante los 90 minutos que dura el partido. Esto también supone que las carreras se desarrollen en pocos años y tengan un retiro temprano.

Allí, la reconversión se impone como una necesidad (Bart 2011). Entre el fin de sus años de arbitraje y su entrada en política, Baldassi ocupó un cargo en la AFA, pero volver a dirigir no es una opción y las posibilidades de volver a desempeñarse en alguna función ligada al fútbol son reducidas. De allí que la inversión en política sea mayor.

Seguí con lo de relaciones públicas [en la AFA] un tiempito más. Hasta que ya salió que iba a hacer candidato a diputado y ya no pude hacer más (Baldassi entrevista).

No proyecto tanto mi vida. [...]. El año que viene termina mi mandato y se verá, los cordobeses son los que van a tener que decidir si siguen confiando, si te dan el voto por cuatro años más. Bueno, en eso estamos.[...]. Seguiría, me gusta. Estoy con unos proyectos más para terminar (Baldassi entrevista). 
Ahora bien, los costos de la reconversión también pueden aumentar en un contexto en el que la política partidaria se encuentra desprestigiada y es vista como una actividad moralmente devaluada. Especialmente para aquellos cuyo prestigio y reconocimiento descansa en su personalidad y su rol público.

La política estaba comenzando a ser desprestigiada. Hoy por hoy es mala palabra hablar de diputados, así que me costaba mucho pensar qué iban a decir de mí. Que era corrupta. La verdad que en el momento en que tomé la decisión sufrí muchísimo. Yo había construido durante muchísimos años el hecho de que la gente creyera en mí. Imaginate perder eso (Tunids entrevista).

En definitiva, vemos cómo varían en cada caso los grados de desinversión en la actividad anterior y de inversión en la política en función de la percepción de cada actor de sus posibilidades de mantener o mejorar su posición en la profesión ejercida. Cuando esas posibilidades son fuertes, como en los casos de Tundis y Flores, la entrada en política no supone un alejamiento de su actividad anterior y el riesgo ante un revés en su carrera política queda mejor cubierto. Es decir que su ingreso a la política no es una inversión tan riesgosa.

En el otro extremo, el ingreso de Baldassi en la competencia partidaria supone un distanciamiento mayor con el mundo del fútbol y una apuesta más fuerte y arriesgada por reconvertirse en político, ante la imposibilidad de volver a ser árbitro. Wolff plantea una situación híbrida entre su continuidad como empresario y su separación de las tareas de dirigente comunitario. Sin embargo, la apuesta por la política encuentra también una buena cobertura de riesgo en su actividad privada.

Esta reversibilidad de los pasajes o los desplazamientos entre distintas esferas de actividad nos lleva a subrayar que resulta más productivo pensar que lo que se reconvierte no son los actores, sino sus recursos o capitales bajo reglas específicas y con diferentes costos.

\section{Una doble condición de marginalidad}

La condición de "recién llegados" a la política partidaria -no a la política a secas, como vimos en algunos casos- comporta una condición de marginalidad relativa, que posee un aspecto objetivo, verificable en su rol en el congreso y en las posiciones subordinadas que ocupan, sumado a la marginalidad subjetiva de no concebirse como un político tradicional. En el primer nivel, aun los que tuvieron una gran visibilidad durante la campaña electoral, no ocupan posiciones destacadas o centrales en la vida de sus partidos, ni en el parlamento ni en los principales debates. Las comisiones de las que forman parte y los cargos que tienen allí son un buen indicador de lo antedicho. Como vemos en el Cuadro 1, predominan los cargos de vocales (el más bajo) a excepción de aquellos temas que constituyen el principal tópico de su "representación simbólica", como la tercera edad para Tundis, el deporte para Baldassi y en menor medida los temas de seguridad interior para Wolff -ligados a la trama del caso Nisman- y de derechos humanos y drogadicción para Flores. Además, ninguno fue autoridad de su bloque.

Ahora bien, si nos centramos en las representaciones que tienen acerca de la política y de su lugar en el Congreso, los cuatro se definen por oposición a lo que entienden como un político tradicional. Éste sería el que conoce las roscas, es ventajero, vive del Estado y sólo busca ganar elecciones para perpetuarse en el poder. En este contrapunto revalorizan su posición de llegados "desde afuera" y del aporte que, en ese sentido, estarían en condiciones de realizar.

No me siento política porque no sé las roscas políticas. Yo sé que adentro de la política hay un montón de cosas y a mí me cuesta, como dicen en la jerga de ellos, "rosquear" o consensuar. [...]. No estoy en las reuniones netamente de los 
${ }^{12}$ Clave Única de Identificación Tributaria (CUIT), número de registro de trabajadores autónomos, comercios y empresas ante el fisco argentino.

${ }^{13}$ Impuesto al Valor Agregado. políticos. [...]. Están los que son políticos porque obviamente nacieron para eso. Se quieren forjar un camino para instalarse con ambiciones de qué sé yo de llegar a ser intendente, de llegar a ser gobernador, de llegar a ser presidente, de llegar a tener un cargo en un ministerio. Yo lo único que quiero es lograr mejoras para los jubilados (Tundis entrevista).

Esta contribución aparece vinculada a la actividad profesional ejercida anteriormente y a la "representación simbólica" que cada uno de ellos comporta. Por ello, en tanto periodista especializada en temas previsionales, Tundis está para conseguir mejoras para los jubilados y Wolff, en su calidad de empresario y a diferencia de los políticos tradicionales, conoce la actividad económica y cómo lograr una articulación entre lo público y lo privado.

Yo creo que todos somos políticos, pero no me siento político convencional. No me siento político convencional en cuanto al estereotipo del político que trata de satisfacer a la gente de la política. [...]. Pero sí, me considero un político y me considero de aquellos políticos que venimos a reivindicar la parte buena de la política, se puede ser un buen político o mal político. [...]. El político tradicional o el estereotipo del político tradicional es alguien que utiliza la política para subsistir. Yo no lo necesito, no vengo de la política. [...]. Por eso creo que no soy un político convencional en función de los estereotipos que perduraron en la Argentina durante tantos años que vos ves tipos que ocupan cargos de muchísima responsabilidad en la toma de decisiones respecto al ámbito privado y sin embargo en su puta vida tuvieron un CUIT $^{12}$, o tuvieron que sentarse a hacer un contracto, o tuvieron que pagar el IVA ${ }^{13}$ (Wolff entrevista).

La vinculación con el trabajo anterior y su mantenimiento como fuente de ingresos posibilita ese distanciamiento del tipo de político "que vive del Estado". Pero el "venir de afuera" también puede suponer dificultades, que redundan en desventajas en su rol de diputados. Los usos y costumbres de la vida parlamentaria se aprenden lentamente y con dificultad. Este "amateurismo" de la vida parlamentaria acentúa su posición relativamente marginal en el espacio político.

La desventaja [de "venir de afuera"] es que vos no tenés cintura política. Yo soy muy frontal en esto. A veces prefería callar y no ser tan frontal (Baldassi entrevista).

A mí me costó mucho adaptarme al ritmo de vida del Congreso, porque yo seguía yendo a las 9 de la mañana, estaba allá. Todos los días de lunes a viernes, a no ser que estuviera haciendo alguna actividad en el interior. Y en el Congreso el lunes no hay nadie y el viernes a la tarde tampoco (risas). Pero bueno yo iba (Flores entrevista).

Ahora bien, el venir "de afuera" puede entonces también convertirse en un capital en sí mismo a la hora de negociar en la arena política. En su análisis de la trayectoria de Margaret Thatcher, King (2002) reconocía un tipo de outsider al que denominaba "estratégico". Este es el que vehiculiza su condición de recién llegado como un recurso más para pelear posiciones en la arena.

Yo podía estar en cualquier lado y no me veían como un competidor. Es más, yo te digo que a veces veía que decían "este es medio boludo". Y eso te facilita. No entras en la competencia despiadada que existe. "Bueno, está todo bien, no nos va a joder". Y eso te permite a vos articular unas cosas que de otra manera no lo podes hacer. Lo negativo es que no te tengan en cuenta para donde están las grandes decisiones. En general, yo me perdía toda esa conversación previa hecha en el bar, porque no iba. Todas esas cosas que no quería ir. Tiene esas contras (Flores entrevista).

A pesar de la posibilidad de utilizar la condición de recién llegado a su favor, la distancia mantenida con las dinámicas partidarias y la vida cotidiana de las instituciones políticas refuerza, al menos al comienzo, la marginalidad de sus 
posiciones. Al desconocer buena parte de las reglas informales del juego político, quedan en desventaja frente a los políticos tradicionales.

\section{Recapitulaciones}

Contrariamente a lo que puede suponerse desde cierto sentido común, los ingresos a la política de personas con una trayectoria profesional por fuera de la competencia partidaria pueden darse de distintas formas y no descansan únicamente en atributos individuales. Las maneras de entrar y los modos en que los recursos generados y acumulados en un ámbito pueden valorizarse en otro distinto son complejas y variables.

Primeramente, reconocemos los dos tipos de recursos extrapolíticos al momento de la entrada, el individual y el colectivo. El primero es un capital construido en torno al prestigio personal del actor y a su reconocimiento mediático vinculado a la o las profesiones ejercidas antes de la política y no supone experiencia previa en actividades de representación. El segundo es colectivo en el sentido de que se trata de un reconocimiento originado en los recursos de una organización o colectivo a la que el actor pertenece o perteneció y en donde sí encontramos experiencia previa en actividades de representación política.

Ambos tipos coexisten en toda estructura de capitales, pero uno de los dos generalmente predomina. En los casos analizados en este artículo, gracias a la variabilidad de rasgos que presentan, puede apreciarse con claridad que mientras en las entradas de Tundis y de Baldassi pesa más lo individual, en las de Wolff y Flores tiene mayor importancia lo colectivo. De esta distinción entre tipos de capital predominante se desprenden otras, que podemos sintetizar en el Cuadro 2.

Asimismo, como veíamos en el cuadro 1, quienes tienen un mayor peso de los recursos individuales se encuentran en los primeros puestos de las listas de candidatos $\left(1^{\circ}\right.$ y $\left.3^{\circ}\right)$. Su conocimiento mediático es jugado más enfáticamente como una carta central en la campaña. En los dos perfiles en los que pesa más lo colectivo, los lugares ocupados son más bajos $\left(6^{\circ}\right.$ y $\left.11^{\circ}\right)$ y, al momento de postularse, no era seguro que constituyeran posiciones expectantes, con chances de ingresar a la cámara. En el caso de Flores, para su segundo mandato ya resulta una figura mucho más conocida que en ocasión de la primera candidatura y lo vemos en el puesto $2^{\circ}$ de la lista.

En segundo lugar, si los aspectos arriba mencionados parecen relacionarse con el mayor peso de lo individual o de lo colectivo, los riesgos que asumen al

Cuadro 2 - Tipos de capital extrapolítico

\begin{tabular}{|c|c|c|}
\hline $\begin{array}{l}\text { Tipo de capital extrapolítico } \\
\text { predominante }\end{array}$ & Individual & Colectivo \\
\hline $\begin{array}{l}\text { Experiencia previa en } \\
\text { actividades de representación }\end{array}$ & - & + \\
\hline Conocimiento mediático & + & - \\
\hline Posiciones expectantes & + & - \\
\hline Profesiones típicas & $\begin{array}{l}\text { Periodistas, deportistas, m } \\
\text { undo del espectáculo }\end{array}$ & $\begin{array}{l}\text { Organizaciones patro- } \\
\text { nales, ONG, movimientos } \\
\text { sociales }\end{array}$ \\
\hline Casos analizados & Tundis y Baldassi & Wolff y Flores \\
\hline
\end{tabular}

Fuente: el autor. 
invertir en la política se entienden más cabalmente en función a la posibilidad o no de volver al ámbito profesional del que provienen, que puede variar según la profesión y de acuerdo a la experiencia concreta de cada actor.

Finalmente, podemos señalar una doble condición de marginalidad que constatamos, en un aspecto más objetivo o posicional, a partir de los lugares relativamente subordinados que ocupan en la vida parlamentaria. En un nivel más subjetivo, vinculado a las representaciones, todos se definen por oposición a los "políticos tradicionales" y manifiestan cierto distanciamiento o extrañamiento con el funcionamiento del Congreso e incluso con algunas prácticas políticas corrientes.

Estas conclusiones no resultan generalizables y no proveen una grilla de análisis extrapolable, sino que apuntan a formular hipótesis de trabajo para comprender y explicar el fenómeno.

Las entradas aquí estudiadas han tenido lugar en grandes distritos, en donde parece concentrarse este fenómeno. También se dan en un contexto de fragmentación partidaria que aparece como estructura de posibilidad de los itinerarios presentados. Estos se desarrollaron en el marco de fuerzas de formación reciente, ya sean agrupaciones nuevas o escisiones de otros frentes, en donde las normas y decisiones no dependen tanto de una institucionalidad previa, sino que poseen un carácter flexible gracias al cual los líderes partidarios tienen un gran peso. Así, desde el punto de vista de los selectores -quienes reclutan a los postulantes y arman las listas-, puede pensarse a este tipo de candidatos como una apuesta más económica y efectiva, ya que, en algunos casos, tienen algún grado de popularidad o conocimiento por parte del electorado y no hace falta "instalarlos" durante la campaña. Pero también podemos verlos como una jugada más segura, ya que su ascenso depende de la intervención personal del líder y no están tan dispuestos - por falta de motivación o de conocimiento de las reglas- a hacer su propio juego partidario (Bruère \& Gaxie, 2014). De modo que también puede hipotetizarse que, para los selectores, son candidatos más leales.

Gabriel Levita (glevita@unla.edu.ar) es doctor en Ciencias Sociales y en Estudios Políticos por la Universidad de Buenos Aires y la École des Hautes Études en Sciences Sociales. Es investigador asistente del CONICET en el Instituto de Problemas Nacionales y profesor adjunto en el Departamento de Planificación y Políticas Públicas de la Universidad Nacional de Lanús (UNLa).

\section{Referencias bibliográficas}

Barr, R. R. 2009. Populists, Outsiders, and Anti-Establishment Politics. Party Politics, 15(1), 29-48. DOI: $10.1177 / 1354068808097890$

Bart, C. Le. 2011. Guy Drut, David Douillet, Bernard Laporte?: la conversion de la gloire sportive en ressource politique. Congrès de La Association Française de Sociologie. Estrasburgo.

Bolognesi, B., Perissinotto, R. M., \& Codato, A. 2019. Reclutamiento político en Brasil. Mujeres, negros y partidos en las elecciones federales de 2014. Revista Mexicana de Ciencias Políticas y Sociales, 61(226), 247-268. DOI: 10.1016/S0185-1918(16)30010-1

Boltanski, L. 1973. L'espace positionnel?: multiplicité des positions institutionnelles et habitus. Revue Française de Sociologie, 14(1), 3-26. DOI: 10.2307/3320321

Bourdieu, P. 1981. La représentation politique - éléments pour une théorie politique. Actes de Recherche En Sciences Sociales, 36/37, 3-24. DOI: 10.3406/arss.1981.2105

Bruère, M. H., \& Gaxie, D. 2014. Le recrutement extra-parlementaire des ministres sous la Ve République. In J.-P. Derosier \& G. Sacriste (Eds.) L'État, le Droit, le Politique Mélanges en l'honneur de Jean-Claude Colliard Paris: Dalloz, pp. 339-349.

Bunker, K., \& Navia, P. 2013. Latin American Political Outsiders, Revisited: The Case of Marco Enríquez-Ominami in Chile, 2009. Journal of Politics in Latin America, 5(2), 3-35. DOI: 10.1177/1866802X1300500201

Canelo, P. 2011. Acerca de la construcción de carreras políticas en la Argentina. Los senadores nacionales en 1973, 1983 y 1989. Polhis, 4(7), 140-153. 
Canelo, P. 2019. Ministros políticos y ministros técnicos. La cuestión de la Defensa en la Argentina desde 1966 a la actualidad. In P. Canelo y M. Heredia (Comps.), Los puentes y las puertas. Las fronteras de la política argentina a través de sus elites. San Martín: UNSAM Edita, pp. 157-180.

Carreras, M. 2012. The Rise of Outsiders in Latin America, 1980-2010: An Institutionalist Perspective. Comparative Political Studies, 45(12), 1451-1482. DOI: 10.1177/0010414012445753

Carreras, M. 2013. Presidentes outsiders y ministros neófitos: un análisis a través del ejemplo de Fujimori. América Latina Hoy, 64, 95-118.

Cheresky, I. 2006. La Política Después de los Partidos. In I. Cheresky (Ed.), La Política Después de los Partidos (Prometeo, pp. 11-23). Buenos Aires.

Cucchetti, H. 2010. Combatientes de Perón, herederos de Cristo. Peronismo, religión secular y organizaciones de cuadros (Prometeo). Buenos Aires.

Dogan, M. 1998. Les professions propices à la carrière politique. Osmoses, filières et viviers. In M. Offerlé (Ed.), La profession politique XIXe-XXe siecles Paris: Belin, pp. 171-199.

Donatello, L. M., \& Levita, G. 2017. ¿Renovación de las elites o renovación de las élites políticas? Los diputados outsiders en los países del Mercosur (2003-2015). Revista de Investigaciones Políticas y Sociológicas, 16(2), 45-64. DOI: 10.15304/rips.16.2.4146

Fillieule, O. 2001. Propositions pour une analyse processuelle de l'engagement individuel. Revue Française de Science Politique, 51(1), 199-215. DOI: 10.3917/rfsp.511.0199

Gaxie, D. 2002. Appréhensions du politique et mobilisations des expériences sociales. Revue Française de Science Politique, 52(2-3), 145-178. DOI: 10.3406/rfsp.2002.403705

Gaxie, D. 2004. La democracia representativa. Santiago de Chile: LOM Ediciones.

Gené, M. 2019. Ministerios políticos del gabinete nacional: perfiles y puertas de entrada de sus elites en democracia. In P. Canelo y M. Heredia, Los puentes y las puertas. Las fronteras de la política argentina a través de sus elites. San Martín: UNSAM Edita, pp. 209-233.

Giorgi, G. I. 2014. Los Factores "Extrapolíticos” de la Carrera Política: Una Aproximación a las Sociabilidades de los ministros de la nación en la Argentina (1854-2011). Política. Revista de Ciencia Política, 52(2), 243-275. DOI: 10.5354/0716-1077.2015.36158

Glaser, B. G., \& Anselm L, S. 1967. The Discovery of Grounded Theory Strategies for Qualitative Research Chicago: Aldine.

Heredia, M. Gené, M., \& Perelmiter, L. 2012. Hacia una socio-historia del Gabinete Nacional. Polhis, 5(9), $284-290$.

Huitt, R. K. 1961. The Outsider in the Senate: An Alternative Role. The American Political Science Review, 55(3), 566-575. DOI: $10.1017 / \mathrm{S} 0003055400125195$

Joignant, A. 2012. Habitus, campo y capital: Elementos para una teoría general del capital político. Revista Mexicana de Sociología, 74(4), 587-618.

Junior, J. A. da S., \& Filho, D. B. F. 2012. Marolas ou tsunamis?: o impacto das ondas de renovação sobre a profissionalização dos deputados federais (1999-2003). Revista de Sociologia e Política, 20(42), 199-212. DOI: 10.1590/S0104-44782012000200014

King, A. 2002. The Outsider as Political Leader: The Case of Margaret Thatcher. British Journal of Political Science, 32(3), 435-454. DOI: $10.1017 /$ S0007123402000182

Landau, M. 2015. Campo político y elencos legislativos en la Ciudad de Buenos Aires (1997- 2011): un análisis sobre la renovación legislativa y política local. Congreso Internacional Élites y Liderazgo En Tiempos de Cambio. Salamanca.

Lascurain, M. C. 2018. Acerca de las élites gubernamentales subnacionales. Los gobernadores y vicegobernadores peronistas de Santa Fe, Argentina (1983-2007). Revista Mexicana de Ciencias Políticas y Sociales, 63(233), 307-332. DOI: 10.22201/fcpys.2448492xe.2018.233.5782

Lévêque, S. 1996. «L'entrée en politique». Bibliographie sur les conditions sociales de la professionnalisation et de la «réussite» politique en France. Politix, 9(35), 171-187.

Levita, G. 2015. La política como profesión: perfiles y tipos de trayectorias de los senadores argentinos. Telos. Revista de Estudios Interdisciplinarios En Ciencias Sociales, 17(1), 38-57.

Linz, J. J. 1994. Presidential or parliamentary democracy: Does it make a difference?. In J. J. Linz \& A. Valenzuela (Eds.), The failure of presidential democracy Baltimore: Johns Hopk, pp. 3-88.

Manin, B. 1998. No Los principios del gobierno representativo Madrid: Alianza.

Massetti, A. 2011. De los movimientos sociales a las urnas: un aporte para el análisis de las transformaciones políticas en nuestro país en comparación con la región. In G. Pérez, O. Aelo, \& G. Salerno (Eds.), Todo aquel fulgor. La política argentina después del neoliberalismo Buenos Aires: Nueva Tril, pp. 53-66.

Mathieu, L. 2007. L'espace des mouvements sociaux. Politix, 77(1), 131-151.

Medina, J. M. A. 2004. La muerte y resurrección de la representación política. México: Fondo de Cultura Económica.

Mellado, V. 2016. La representación política en cuestión. Trayectorias de los legisladores de Mendoza en el largo plazo (1946-1999). In S. Mauro, V. O. de Rozas, \& M. P. V. Narvaja (Eds.), Política Subnacional en Argentina. Enfoques y problemas Buenos Aires: CEAP-Honor, pp. 143-177.

Mustapic, A. M. 2013. Los partidos políticos en la Argentina: condiciones y oportunidades de su fragmentación. In C. H. Acuña (Ed.), ¿Cuánto importan las instituciones? Gobierno, Estado y actores en la política argentina. Buenos Aires: Siglo XXI, pp. 249-290.

Novaro, M. 2000. Representación y liderazgo en las democracias contemporáneas Rosario: Homo Sapie. 
Offerlé, M. 1996. Entrées en politique. Politix, 9(35), 3-5.

Offerlé, M. 2004. Los partidos politicos. Santiago de Chile: LOM Ediciones.

Offerlé, M. 2011. Los oficios, la profesión y la vocación de la política. PolHis, 1(7), 84-98.

Perelmiter, L. 2012. . La constitución de una autoridad plebeya. El ministerio "de la pobreza" en la Argentina reciente. Polhis, 5(9), 309-318.

Rodrigo, C. 2014. Actores, relaciones y escaños. Apuntes para el estudio de las carreras políticas de los diputados nacionales por la Provincia de Buenos Aires. Estudios Digital, 32, 135-148. DOI: 10.31050/1852.1568.n32.11587

Rozas, V. O. de. 2011. Las formas de reclutamiento del personal político, una vía de entrada al estudio del régimen político provincial. Santiago del Estero (1999-2009). Revista Perspectivas de Políticas Públicas, Universidad Nacional de Lanús, 1(1), 133-159. DOI: 10.18294/rppp.2011.647

Santos, A. M. Dos. 1997. Nas Fronteiras do campo político. Raposas e outsiders no congresso nacional. Revista Brasileira de Ciências Sociais, 12(33), 87-101.

Sawicki, F. 1999. Classer les hommes politiques. In M. Offerlé (Ed.), La profession politique, XIXe-XXe siècles Paris: Belin, pp. 135-170.

Sosa, P. 2019. Los dirigentes del Frente para la Victoria Santacruceña: una elite de militantes (1988-2003). In P. Canelo y M. Heredia, Los puentes y las puertas. Las fronteras de la política argentina a través de sus elites. San Martín: UNSAM Edita, pp. 109-120.

Tissot, S. 2004. Les reconversions militantes. In M. H. L. S. Tissot, C. Gaubert (Eds.), Reconversions militantes Paris: Pulim, pp. 9-17.

Torre, J. C. 2003. Los huérfanos de la política de partidos. Sobre los alcances y la naturaleza de la crisis de representación partidaria. Desarrollo Económico, 42(168), 647-665.

Torre, J. C. 2017. Los huérfanos de la política de partidos revisited. Revista SAAP, 11(2), 241-249.

Vázquez, M. 2014. «Militar la gestión»: una aproximación a las relaciones entre activismo y trabajo en el Estado en Argentina en las gestiones de gobierno de Cristina Fernández de Kirchner. Apuntes, 41(74), 71-102. DOI: 10.21678/apuntes.74.703

Vommaro, G. 2017. La larga marcha de Cambiemos. La construcción silenciosa de un proyecto de poder . Buenos Aires: Siglo XXI.

Vommaro, G., \& Armesto, M. 2015. ¿Nuevos políticos en el partido, viejos políticos en las listas? Reclutamiento partidario y división del trabajo político en PRO, en la Ciudad Autónoma de Buenos Aires. Pasado Abierto. Revista Del CEHis, 2 , 110-132.

Weber, M. 2007. La política como profesión. In La ciencia como profesión - La política como profesión . Madrid: Austral, pp. 85-152.

\section{Otras fuentes}

BH., 2016. ¿Qué cobrás? Mi autobiografía. Buenos Aires: Planeta.

EL CRONISTA. 2015. Quién es Toty Flores, el vice de Carrió. Buenos Aires, 10 jun. Disponible en: https://www.cronista.com/economiapolitica/Quien-es-Toty-Flores-el-vice-de-Carrio-20150610-0130.html. Accedido en: 13 dic. 2018.

FDL. 2011. Directorio de Legisladores. Disponible en: http://directorio.directoriolegislativo.org/. Accedido en: 13 dic. 2018.

HB. 2012. "A varios árbitros les falta ser futboleros", 2012. El Gráfico. Buenos Aires: 9 feb. Disponible en: http://www.elgrafico.com.ar/2012/02/09/C-4067-hector-baldassi-a-varios-arbitros-les-falta-ser-futboleros.php. Accedido en: 13 dic. 2018.

HB. 2016. Entrevista a Héctor Badassi.

HL. 2016. Entrevista a Héctor Flores.

LA NACION. 2015. La historia íntima de Toty Flores, el vice de Carrió que vive en una villa. La Nación. Buenos Aires, 1 jul. Disponible en: https://www.lanacion.com.ar/1806381-la-historia-in-

tima-de-toty-flores-el-vice-de-carrio-que-vive-en-una-villa. Accedido en: 13 dic. 2018.

MT. 2014. Diputada Mirta Tundis. Disponible en: https://es-la.facebook.com/tundismirta/. Accedido en: 13 dic. 2018.

MT. 2016. Entrevista a Mirta Tundis.

WH. 2016. Entrevista a Waldo Wolff.

WW. 2015. Waldo Wolff ¿Quién soy? Disponible en: http://www.waldowolff.com.ar/\#!/quiensoy. Accedido en: 17 sep. 2017. 


\section{Getting into the Argentine Chamber of Deputies "from outside": resources, investments and marginalities}

ABSTRACT Introduction: This article looks, from a qualitative political sociology, into the social trajectories and entries to politics of a group of Argentine national congressmen that were elected after 2007, having accumulated resources and recognition in other professional fields. Materials and Methods: We reconstruct their professional trajectories, recruitments, entries to politics and arrivals to the Lower Chamber from in-depth interviews and secondary sources. The cases were chosen based on the possibilities each of them offers to show a variety of socio-professional origins and kinds of political capitals. Results: The analysis is centered in three dimensions: a) the reconversion of the resources accumulated in the previous professions into political capital; b) the entrance to political competition in terms of investments and risk; and c) the subordinated position of these actors when they enter to the Congress. We show how: a) entering "from outside" can be made with two types of resources, individual and collective; b) risks assumed are linked to the chances of returning to the professional field they were in before; and c) there is a double marginal condition, for they are in subordinated positions in Congress life and feel aliens regarding the Congress rules. Discussion: The article contributes to the knowledge on contemporary Argentine political elites and, especially, on those who enter to Congress "from outside" by using French political sociology's conceptualization on the structures of resources these actors have while they enter politics. Thus, it allows to understand the social conditions of political recruitment beyond institutional variables.

KEYWORDS: Political Elites; Entering Politics; Political Trajectories; National Congressmen; Argentina.

This is an Open Access article distributed under the terms of the Creative Commons Attribution Non-Commercial License which permits unrestricted non-commercial use, distribution, and reproduction in any medium provided the original work is properly cited.

A produção desse manuscrito foi viabilizada através do patrocínio fornecido pelo Centro Universitário Internacional Uninter à Revista de Sociologia e Política. 